\title{
Linhagens de frangos caipiras criadas em sistema semi-intensivo em região de clima quente
}

\author{
Adélio Nunes Dias ${ }^{(1)}$, Mônica Patrícia Maciel(2), Auriclécia Lopes de Oliveira Aiura(2), \\ Cláudio Luiz Correa Arouca ${ }^{(2)}$, Daiane Batista Silva ${ }^{(2)}$ e Vítor Hugo Santana de Moura ${ }^{(2)}$
}

\begin{abstract}
(1)Universidade Federal Rural do Rio de Janeiro, Instituto de Zootecnia, Rodovia BR 465, Km 07, Seropédica, Caixa Postal 74.580, CEP 23890-000 Rio de Janeiro, RJ, Brasil. E-mail: adelionunes7@yahoo.com.br (2)Universidade Estadual de Montes Claros, Departamento de Ciências Agrárias, Avenida Reinaldo Viana, no 2630, Bico da Pedra, Caixa Postal 91, CEP 39440-000, Janaúba, MG, Brasil. E-mail: monicapmaciel@hotmail.com, aurilopes@yahoo.com.br, claudioarouca@yahoo.com.br, daianebatistas88@yahoo.com.br, santmoura02@gmail.com
\end{abstract}

Resumo - O objetivo deste trabalho foi avaliar o desempenho, as variáveis fisiológicas e o comportamento de cinco linhagens de frangos de corte caipiras criadas em sistema semi-intensivo, em região de clima quente. Utilizaram-se 400 aves, com idade inicial de 35 dias, em cinco tratamentos representados pelas linhagens caipiras: Pescoço Pelado, Vermelho Pesadão, Carijó, Master Griss e Colorido. Utilizou-se um delineamento inteiramente casualizado, com quatro repetições e 20 parcelas. Avaliaram-se desempenho (ganho de peso, consumo de ração e conversão alimentar), temperatura retal, frequência respiratória e comportamento. Não houve diferenças significativas entre as linhagens para desempenho e variáveis fisiológicas. Todas as linhagens apresentaram desconforto térmico e, com base nos comportamentos observados, notou-se uma adaptação à alta temperatura. As linhagens Pescoço Pelado, Vermelho Pesadão, Carijó, Master Griss e Colorido podem ser utilizadas para a criação em sistema semi-intensivo, em região de clima quente.

Termos para indexação: ambiência, avicultura alternativa, comportamento, desempenho.

\section{Strains of free-range broilers reared in semi-intensive system in a hot climate region}

\begin{abstract}
The objective of this work was to evaluate the performance, physiological traits, and behavior of five lines of free-range broilers reared in a semi-intensive system in hot climate region. Four hundred birds, with an initial age of 35 days, were used in five treatments represented by the free-range lines: Pescoço Pelado, Vermelho Pesadão, Carijó, Master Griss, and Colorido lines. A completely randomized design with four replicates and 20 plots was used. Performance (weight gain, feed intake, and feed conversion), rectal temperature, respiratory frequency, and behavior were evaluated. There were no significant differences between the lines for performance and physiological variables. All lines showed thermal discomfort and, based on their behaviors, an adaptation trend to hot climate was noted. The Pescoço Pelado, Vermelho Pesadão, Carijó, Master Griss, and Colorido lines can be reared in semi-intensive systems in hot climate region.
\end{abstract}

Index terms: ambience, alternative poultry farming, behavior, performance.

\section{Introdução}

As aves caipiras criadas no sistema semi-intensivo apresentam uma carcaça diferenciada, com menor quantidade de gordura, além de textura e sabor peculiares (Takahashi et al., 2006). Essas características estão relacionadas ao tipo de ave utilizada (linhagens específicas de crescimento lento); maior atividade de pastejo e movimentação; consumo de forragens (fontes de pigmentos carotenoides), insetos, minhocas, etc.; e idade de abate igual ou superior a 85 dias (Savino et al., 2007; Faria et al., 2009). O bem-estar na produção de aves está diretamente relacionado com o sistema de criação (Moura et al., 2010; Fraser et al., 2013). O sistema de semiconfinamento permite que as aves tenham livre acesso às áreas de pastejo, para que estas expressem seus comportamentos naturais, resultando em diferenças particulares na qualidade da carne, quando comparadas com as aves criadas confinadas (Fanatico et al., 2007; Mikulski et al., 2011). Por outro lado, as condições ambientais podem interferir no bem-estar, saúde e comportamento das aves, podendo se refletir em suas características fisiológicas e produtivas (Fouad et al., 2008; Tan et al., 2010). 
Uma das variáveis ambientais mais importantes que pode interferir no ambiente de criação das aves é a temperatura, responsável por afetar diretamente os animais. De forma geral, as aves em estresse calórico procuram manter sua homeostase, apresentando mudanças comportamentais como: diminuição da atividade locomotora, asas afastadas do corpo e aumento da ofegação (Mack et al., 2013). Além desses comportamentos, aumentam o consumo de água e o consumo de ração, o que interfere diretamente na conversão alimentar, taxa de crescimento, rendimento de carcaça e qualidade da carne (Lara \&Rostagno, 2013).

As respostas ao estresse calórico podem ser diferentes, dependendo da linhagem utilizada ( $\mathrm{Lu}$ et al., 2007; Soleimani et al., 2011). Segundo Cordeiro et al. (2014), em regiões de altas temperaturas, é importante a escolha de linhagens adaptadas e resistentes ao estresse térmico para produção de carne de qualidade com menores custos de produção e maior eficiência produtiva e, ao mesmo tempo, promover o bem-estar aos animais.

Existe escassez na literatura de pesquisas relativas à comparação entre linhagens caipiras criadas em regiões de altas temperaturas, e os pouco dados encontrados foram obtidos por meio de trabalhos práticos e de observação a campo.

O objetivo deste trabalho foi avaliar o desempenho, as variáveis fisiológicas e o comportamento de cinco linhagens de frangos de corte caipiras criadas em sistema semi-intensivo em região de clima quente.

\section{Material e Métodos}

O experimento foi realizado na cidade de Janaúba, norte do Estado de Minas Gerais, a qual possui latitude de $15^{\circ} 47^{\prime} 50^{\prime \prime} \mathrm{S}$ e longitude de $43^{\circ} 18^{\prime} 31^{\prime \prime} \mathrm{W}$ e altitude de 516 m. O clima, segundo a classificação de Köppen, é do tipo Aw, tropical chuvoso, de savana, com inverno seco.

A área experimental era composta de 20 piquetes de $30 \mathrm{~m}^{2}$. Cada piquete continha um abrigo de madeira coberto com telhas de fibrocimento e telado em suas laterais, com medidas de 1,8 m de pé-direito x $1,50 \mathrm{~m}$ de comprimento x 2,0 m de largura.

Foram utilizadas 400 aves de cinco linhagens caipiras melhoradas, e o delineamento experimental utilizado foi o inteiramente casualizado, com 5 tratamentos e 4 repetições, totalizando 20 unidades experimentais.
As linhagens constituíram os tratamentos, sendo: Pescoço Pelado (Label Rouge), Vermelho Pesadão, Carijó, Master Griss e Colorido.

As aves foram adquiridas com 28 dias de vida e foi mensurada a massa corporal para formar unidades experimentais homogêneas. Utilizou-se um período de 7 dias de adaptação antes de proceder à coleta de dados. Os animais foram presos nos abrigos durante o período da noite para evitar ataques de predadores, e durante o dia foram soltos, tendo tido livre acesso à área de pastejo com capim Tifton 85 (Cynodon dactylon) e ao interior do abrigo. A partir dos 35 dias de idade, iniciou-se o período experimental.

O programa alimentar foi dividido em períodos de crescimento (35 a 61 dias) e terminação (62 a 85 dias), tendo sido utilizadas rações comerciais contendo energia metabolizável de 3.150 e $3.200 \mathrm{kcal} \mathrm{kg}^{-1}$ e proteína bruta de 18 e $17,3 \%$, respectivamente. $\mathrm{O}$ arraçoamento foi feito uma vez ao dia.

Foram realizadas observações e registros das variáveis ambientais e de comportamento das aves durante 35 dias nos horários de $8 \mathrm{~h}, 10 \mathrm{~h}, 12 \mathrm{~h}, 14 \mathrm{~h}$ e 16h. Para as variáveis ambientais, foram distribuídos na área de pastejo 12 globos negros (para mensurar a temperatura de globo negro), que foram confeccionados com globos de plástico de $15 \mathrm{~cm}$ de diâmetro, pintados com tinta preta fosca, e no interior de cada um deles foi colocado um termômetro de mercúrio (-10 a + $110^{\circ} \mathrm{C}$; Incoterm, Porto Alegre, RS). Para medição das temperaturas de bulbo seco e bulbo úmido, 20 termohigrômetros (-10 a $50^{\circ} \mathrm{C}$; Incoterm, Porto Alegre, RS) foram colocados dentro dos abrigos na altura das aves. A velocidade do vento foi registrada com o auxílio de um anemômetro modelo Rotor Axial Kestrel 4500 (Kestrel, Birmingham, MI, USA). As variáveis ambientais foram utilizadas para calcular a pressão parcial de vapor e a carga térmica radiante (CTR) conforme Silva (2000), e o índice de temperatura de globo negro e umidade (ITGU) de acordo com Buffington et al. (1981).

A avaliação de comportamento foi realizada utilizando-se o método Scan Instantâneo (Martin \& Bateson, 1986) durante 2 minutos por parcela, tendo sido observados e registrados os comportamentos apresentado pelas aves, por método adaptado de Gonçalves (2012) (Tabela 1).

As variáveis fisiológicas avaliadas foram temperatura retal e frequência respiratória. Foram 
selecionadas aleatoriamente 3 aves por parcela, e as medidas foram realizadas a cada sete dias nos horários de $9 \mathrm{~h}$ e $15 \mathrm{~h}$. A temperatura retal foi medida utilizando-se um termômetro clínico veterinário $\left(32^{\circ} \mathrm{C}\right.$ a $43,9^{\circ} \mathrm{C}$, Incoterm), com precisão de $\pm 0,1^{\circ} \mathrm{C}$, inserido através da cloaca da ave até que fosse atingida temperatura constante. A frequência respiratória foi aferida por meio da contagem de movimentos abdominais realizados pela ave, por 1 minuto. A avaliação de desempenho foi realizada conforme o consumo de ração, ganho de massa corporal e conversão alimentar. $\mathrm{O}$ arraçoamento foi realizado diariamente, tendo sido anotadas as quantidades servidas na semana por parcela, e no final de 7 dias eram pesadas as sobras de ração. A massa corporal das aves foi obtida semanalmente. O número de aves mortas foi anotado para cálculo da porcentagem de mortalidade.

Após o final do experimento, os dados de desempenho e das variáveis fisiológicas foram submetidos à análise de variância e suas médias foram comparadas pelo teste de Scott-Knott, a de 5\% de probabilidade, utilizando-se o programa estatístico Sisvar (Universidade Federal de Lavras, Lavras, MG). As variáveis comportamentais foram analisadas pelo teste não paramétrico de KruskalWallis, por meio do software SAS (SAS Institute Inc., Cary, NC, EUA).

\section{Resultados e Discussão}

Durante a fase experimental, foram encontrados os valores para ITGU máximo e mínimo de 95 e 86, respectivamente, e CTR máxima e mínima de 770 e $670 \mathrm{~W} \mathrm{~m}^{-2}$, respectivamente. Estes valores são considerados como característicos de ambiente fora da faixa de conforto térmico para as aves, pois, segundo Medeiros et al. (2005), a CTR e o ITGU não podem ultrapassar valores de 500,3 e $77 \mathrm{~W} \mathrm{~m}^{-2}$, respectivamente.

As variáveis de desempenho (massa corporal final, ganho de massa corporal, consumo de ração e conversão alimentar) não diferiram entre as linhagens avaliadas (Tabela 2).

Madeira et al. (2010) não encontraram diferenças no desempenho entre as linhagens Master Griss, Label Rouge e Vermelho Pesadão, porém, observaram valores superiores aos desta pesquisa para ganho de massa corporal, tendo sido: 3,$13 ; 2,62$ e $3,21 \mathrm{~kg}$ e consumo de ração de 10,76; 8,70 e 10,76 kg, respectivamente.

Del Castilho et al. (2013) encontraram resultados diferentes dos deste trabalho, em que o ganho de massa corporal foi maior para as linhagens Vermelho Pesadão e Label Rouge. Quanto à conversão alimentar, as linhagens não diferiram umas das outras.

Ao avaliar o desempenho de linhagens caipiras, entre elas Carijó, Vermelho Pesadão e Label Rouge,

Tabela 1. Etograma comportamental para frangos de corte.

\begin{tabular}{|c|c|}
\hline Comportamento & Descrição \\
\hline Sentado & Caracterizado quando o corpo das aves está em contato com o solo, piso ou cama. \\
\hline Comendo & Consumindo ou procurando ração no comedouro. \\
\hline Bebendo & Consumindo água no bebedouro. \\
\hline Forrageando & Consumindo e/ou bicando o substrato vegetal ou o solo na área do piquete. \\
\hline Explorando penas & Explorando as penas com o bico, tanto para manutenção, quanto para investigação. \\
\hline Bicagem não agressiva & $\begin{array}{l}\text { Bicando levemente outras aves, geralmente na região inferior ventral do pescoço, dorso, base e ponta da cauda ou } \\
\text { abdômen. }\end{array}$ \\
\hline Bicagem agressiva & $\begin{array}{l}\text { Bicagem forte de outra ave, provocando reação agressiva ou defensiva, geralmente direcionada à região superior da } \\
\text { cabeça e crista ou à região inferior dorsal do pescoço. }\end{array}$ \\
\hline Movimentos de desconforto & $\begin{array}{l}\text { Ave ofegando e apresentando uma das seguintes posições: sentada, ou em pé com asas abertas e afastadas do corpo, ou } \\
\text { sentada com asas e pernas esticadas e afastadas do corpo. }\end{array}$ \\
\hline Ciscando & Quando a ave explora seu território com seus pés e bico, direcionados ao piso. \\
\hline Banho de areia & Quando a ave revolve-se no substrato da cama ou no solo na área do piquete, espalhando-o pelo corpo. \\
\hline Ócio & Ave parada, sem movimentação expressiva. \\
\hline
\end{tabular}

Fonte: adaptado de Gonçalves (2012). 
Veloso et al. (2014) verificaram diferenças entre as linhagens para o ganho de massa corporal, em que a linhagem Label Rouge diferiu das demais, com menor valor. Esses autores verificaram maiores valores de consumo de ração $(10,6 ; 10,9$; e $10,3 \mathrm{~kg}$, respectivamente) e ganho de massa corporal $(3,82$; 3,88 ; e $3,60 \mathrm{~kg}$, respectivamente) do que os observados no presente trabalho.

Concordando com os resultados desta pesquisa em relação à massa corporal final, Madeira et al. (2010) também não observaram diferenças entre as linhagens Master Griss, Label Rouge e Vermelho Pesadão. Porém, a massa corporal final encontrada pelos autores citados passou de $3 \mathrm{~kg}$ para as linhagens avaliadas, enquanto o observado nesta pesquisa chegou, no máximo, a 2,27 kg para a linhagem Carijó.

Existem diferenças de valores de ganho de massa corporal e consumo de ração, e os valores observados neste trabalho foram menores em relação à literatura. Isso pode ter ocorrido em virtude do manejo do arraçoamento, pois as aves eram alimentadas somente uma vez ao dia, com porções iguais para cada ave e, nos demais trabalhos, o arraçoamento foi feito à vontade.

$\mathrm{O}$ arraçoamento à vontade deve ser observado com critério pelos criadores de frangos caipiras, pois se este for adotado, as aves podem apresentar peso corporal excessivo. Segundo Veloso et al. (2014), isso pode prejudicar a comercialização destes animais, pois o consumidor brasileiro de frango caipira demanda principalmente o frango inteiro e uma carcaça não muito grande. O arraçoamento à vontade também pode fazer com que a ave não sinta a necessidade de procurar o alimento na área de pastejo disponível, podendo não adquirir atributos importantes esperados nestes animais, como coloração mais acentuada da carcaça e firmeza e sabor característicos da carne (Mikulski et al., 2011).

As variáveis temperatura retal e frequência respiratória não diferiram entre as linhagens (Tabela 3). Silva et al. (2001) submeteram diferentes linhagens de frango a estresse térmico e observaram maior temperatura retal de aves de linhagem com empenamento completo comparadas àquelas de empenamento reduzido (Pescoço Pelado). Os autores relatam que a menor temperatura retal apresentada por esta linhagem, durante o período de estresse, é resultante de um melhor mecanismo de termorregulação da temperatura corporal apresentado por essas aves. Porém, concordando com os resultados da presente pesquisa, Cordeiro et al. (2014) não verificaram diferenças na temperatura retal quando compararam as linhagens Pesadão e Pescoço Pelado.

As médias de temperatura retal encontradas nesta pesquisa estão de acordo com a literatura (Silva et al., 2006; Nazareno et al., 2011; Cordeiro et al., 2014), variando entre 41 e $42^{\circ} \mathrm{C}$, o que demonstra que, apesar da alta temperatura ambiente, os animais conseguiram manter sua temperatura interna, o que é uma evidencia de que todas as linhagens se mostraram adaptadas ao ambiente.

Segundo Medeiros et al. (2005), a CTR e o ITGU não podem ultrapassar valores de 500,3 e $77 \mathrm{~W} \mathrm{~m}^{-2}$, respectivamente. No presente trabalho, os valores de ITGU máximo e mínimo foram de 95 e 86 ,

Tabela 2. Massa corporal final (MCF), consumo médio de ração (CR), ganho médio massa corporal (GMC) e conversão alimentar (CA) de acordo com as linhagens aos 85 dias.

\begin{tabular}{|c|c|c|c|c|c|c|c|}
\hline \multirow[t]{2}{*}{ Variável } & \multicolumn{5}{|c|}{ Linhagem } & \multirow{2}{*}{$\begin{array}{l}\text { CV } \\
(\%)\end{array}$} & \multirow[t]{2}{*}{$\mathrm{F}$} \\
\hline & Label Rouge & Vermelho Pesadão & Carijó & Master Griss & Colorido & & \\
\hline $\mathrm{MCF}(\mathrm{Kg})$ & 2,26 & 2,24 & 2,29 & 2,20 & 2,26 & 2,15 & 0,134 \\
\hline CR (Kg) & 5,05 & 5,14 & 5,17 & 5,17 & 5,22 & 2,23 & 0,341 \\
\hline GMC (Kg) & 1,78 & 1,76 & 1,77 & 1,72 & 1,81 & 3,24 & 0,315 \\
\hline $\mathrm{CA}$ & 2,84 & 2,94 & 2,90 & 3,00 & 2,88 & 3,69 & 0,322 \\
\hline
\end{tabular}

Tabela 3. Valores médios de temperatura retal (TR) e frequência respiratória (FR) de acordo com as linhagens.

\begin{tabular}{|c|c|c|c|c|c|c|c|}
\hline \multirow[t]{2}{*}{ Variável } & \multicolumn{5}{|c|}{ Linhagem } & \multirow{2}{*}{$\begin{array}{l}\text { CV } \\
(\%)\end{array}$} & \multirow[t]{2}{*}{$\mathrm{F}$} \\
\hline & Label Rouge & Vermelho Pesadão & Carijó & Master Griss & Colorido & & \\
\hline $\mathrm{TR}\left({ }^{\circ} \mathrm{C}\right)$ & 41,4 & 41,4 & 40,9 & 40,9 & 41,5 & 1,28 & 0,560 \\
\hline FR & 50,8 & 48,4 & 52,3 & 52,9 & 51,3 & 7,32 & 0,510 \\
\hline
\end{tabular}


respectivamente, e carga térmica radiante (CTR) máxima e mínima de 770 e $670 \mathrm{~W} \mathrm{~m}^{-2}$, respectivamente. Estes valores encontrados foram preponderantes para as aves aumentarem a frequência respiratória, utilizando com mais ênfase a troca de calor latente, como corroborado por valores encontrados por Silva et al. (2003), variando de 94 a 115 movimentos por minuto sob altas temperaturas.

Foram observadas diferenças significativas entre as linhagens apenas nos comportamentos: "Bebendo", "Explorando Penas" e "Bicagem Não Agressiva" (Tabela 4). Pereira et al. (2007) afirmam que aves em estresse térmico alteram seu comportamento no intuito de tentar manter a temperatura corporal dentro de limites normais, e os ajustes de comportamento podem ocorrer rapidamente e a um custo menor do que os ajustes fisiológicos.

Para o comportamento "Bebendo", as linhagens Vermelho Pesadão, Carijó e Master Griss foram as que apresentaram maior frequência no consumo de água, não diferindo entre si, enquanto as linhagens Label Rouge e Colorido obtiveram menor consumo, não diferindo uma da outra. Esse menor consumo de água para manutenção da temperatura interna pode ser decorrente da maior adaptação destas linhagens ao clima (Pereira et al., 2007). Contudo, todas as linhagens apresentaram uma maior proporção de aves sentadas, comportamento mais evidente quando as aves estão em desconforto térmico (Santos et al., 2010; Carvalho et al., 2013), caso comum em regiões de clima quente. Isso ocorreu pela dificuldade desse animal de trocar calor por radiação e convecção (Cangar et al., 2008), tendo sido necessário o contato com uma superfície de menor temperatura, como o solo sombreado, para uma troca mais efetiva de calor por condução.
A variável "Explorando Penas" é uma característica que demonstra que as aves estão em conforto, e apresentam um comportamento de repertório natural (Barbosa Filho et al., 2007; Santos et al., 2010). Nesta pesquisa, observou-se pouca ocorrência desse comportamento, provavelmente em virtude das condições térmicas acima das consideradas como favoráveis às aves. Entre as linhagens, a Label Rouge apresentou o menor número de animais que expressaram este comportamento, não tendo diferido das linhagens Carijó e Master Griss; contudo, tal diferença pode ocorrer pela diferença natural de cada linhagem.

Além das condições climáticas, a exploração de penas parece ser muito influenciada pelo enriquecimento ambiental. Em pesquisa desenvolvida por Santos et al. (2010), com frangos caipiras também em região quente, quando os animais tiveram acesso a piquete com poleiro e sombreamento, o comportamento "Explorando Penas" foi exibido três vezes mais do que quando havia apenas acesso ao piquete.

Quanto ao comportamento "Bicagem Não Agressiva", poucas aves o fizeram, e a linhagem Master Griss diferenciou-se das demais, tendo apresentado maior quantidade de animais que realizaram esse comportamento. Segundo Dixon et al. (2010), a bicagem de penas é considerada um redirecionamento do comportamento de bicagem de alimentos, e o fato de as aves terem espaço e substratos para serem explorados diminui a expressão desse comportamento (Santos et al., 2010).

Foram observadas diferenças entre os comportamentos das linhagens nos diferentes horários do dia com exceção dos denominados "Bebendo" e "Banho de Areia" (Tabela 5).

Os horários das $8 \mathrm{~h}$ e $16 \mathrm{~h}$ foram os que apresentaram menor número de aves sentadas, porém, no horário das

Tabela 4. Comportamentos das aves no período experimental de acordo com as linhagens ${ }^{(1)}$.

\begin{tabular}{|c|c|c|c|c|c|c|c|c|c|c|c|}
\hline \multirow[t]{2}{*}{ Tratamento } & \multicolumn{11}{|c|}{ Comportamento $^{(2)}$} \\
\hline & Sentado & Comendo & Bebendo & $\begin{array}{l}\text { Forra- } \\
\text { geando }\end{array}$ & $\begin{array}{l}\text { Explorando } \\
\text { penas }\end{array}$ & $\begin{array}{c}\text { Bicagem } \\
\text { NA }\end{array}$ & $\begin{array}{l}\text { Bicagem } \\
\text { agressiva }\end{array}$ & MD & Ciscando & $\begin{array}{c}\text { Banho } \\
\text { de areia }\end{array}$ & Ócio \\
\hline Label Rouge & 7,5 & 1,4 & $1,0 \mathrm{~b}$ & 1,0 & $1,2 \mathrm{~b}$ & $0,08 \mathrm{~b}$ & 0,08 & 4,3 & 1,2 & 0,09 & 1,0 \\
\hline Vermelho Pesadão & 7,6 & 1,1 & $1,2 \mathrm{a}$ & 1,0 & $1,6 \mathrm{a}$ & $0,06 \mathrm{~b}$ & 0,03 & 4,6 & 1,3 & 0,08 & 1,1 \\
\hline Carijó & 7,5 & 1,4 & $1,2 \mathrm{a}$ & 0,9 & $1,4 \mathrm{ab}$ & $0,07 b$ & 0,05 & 4,4 & 1,3 & 0,06 & 1,0 \\
\hline Master Griss & 7,2 & 1,6 & $1,2 \mathrm{a}$ & 1,1 & $1,4 \mathrm{ab}$ & $0,14 \mathrm{a}$ & 0,06 & 4,4 & 1,3 & 0,14 & 1,2 \\
\hline Colorido & 7,5 & 1,2 & $1,0 \mathrm{~b}$ & 1,0 & $1,6 \mathrm{a}$ & $0,07 \mathrm{~b}$ & 0,07 & 4,2 & 1,4 & 0,11 & 1,1 \\
\hline
\end{tabular}

${ }^{(1)}$ Médias seguidas de letras diferentes, na mesma coluna, diferem pelo Teste de Kruskal-Wallis a 5\% de probabilidade. ${ }^{(2)} \mathrm{NA}$, não agressiva; MD, movimento de desconforto. 
Tabela 5. Comportamentos das aves no período experimental de acordo com o horário ${ }^{(1)}$.

\begin{tabular}{|c|c|c|c|c|c|c|c|c|c|c|c|}
\hline \multirow{2}{*}{$\begin{array}{l}\text { Horário } \\
\text { (h) }\end{array}$} & \multicolumn{11}{|c|}{ Comportamento $^{(2)}$} \\
\hline & Sentado & Comendo & Bebendo & $\begin{array}{l}\text { Forra- } \\
\text { geando }\end{array}$ & $\begin{array}{l}\text { Explorando } \\
\text { penas }\end{array}$ & $\begin{array}{c}\text { Bicagem } \\
\text { NA }\end{array}$ & $\begin{array}{l}\text { Bicagem } \\
\text { agressiva }\end{array}$ & MD & Ciscando & $\begin{array}{l}\text { Banho } \\
\text { de areia }\end{array}$ & Ócio \\
\hline 8:00 & $8,0 \mathrm{~b}$ & $1,6 \mathrm{a}$ & 1,1 & $2,0 \mathrm{a}$ & $1,9 \mathrm{a}$ & $0,10 \mathrm{a}$ & $0,03 b$ & $0,27 \mathrm{e}$ & $2,6 \mathrm{a}$ & 0,13 & $1,41 \mathrm{a}$ \\
\hline 10:00 & $10,0 \mathrm{a}$ & $1,7 \mathrm{a}$ & 1,1 & $0,4 b$ & $1,4 \mathrm{~b}$ & $0,06 \mathrm{~b}$ & $0,05 b$ & $1,83 \mathrm{~d}$ & $1,4 \mathrm{~b}$ & 0,12 & $1,22 \mathrm{a}$ \\
\hline $12: 00$ & $7,0 \mathrm{c}$ & $1,2 b$ & 1,2 & $0,2 b$ & $1,1 \mathrm{c}$ & $0,07 \mathrm{~b}$ & $0,04 \mathrm{~b}$ & $6,67 \mathrm{~b}$ & $0,7 \mathrm{c}$ & 0,07 & $0,83 \mathrm{c}$ \\
\hline 14:00 & $6,0 \mathrm{~d}$ & $0,9 \mathrm{c}$ & 1,0 & $0,3 b$ & $1,0 \mathrm{c}$ & $0,07 \mathrm{~b}$ & $0,04 \mathrm{~b}$ & $8,23 \mathrm{a}$ & $0,7 \mathrm{c}$ & 0,08 & $0,69 \mathrm{~d}$ \\
\hline $16: 00$ & $5,0 \mathrm{e}$ & $1,2 \mathrm{~b}$ & 1,0 & $2,0 \mathrm{a}$ & $1,8 \mathrm{a}$ & $0,10 \mathrm{a}$ & $0,14 \mathrm{a}$ & $5,00 \mathrm{c}$ & $1,1 \mathrm{~b}$ & 0,09 & $1,16 \mathrm{~b}$ \\
\hline
\end{tabular}

${ }^{(1)}$ Médias seguidas de letras diferentes na mesma coluna diferem pelo Teste de Kruskal-Wallis a $5 \%$ de probabilidade. ${ }^{(2)} \mathrm{NA}$, não agressiva; MD, movimento de desconforto.

16h, apareceram aves com movimentos de desconforto associados. No horário das $10 \mathrm{~h}$, observou-se o maior número de aves sentadas, e pode-se dizer que esse comportamento de contato do corpo com o solo possivelmente estava sendo feito na tentativa de trocar calor por condução para termorregulação (Cangar et al., 2008). Houve uma incidência maior de aves em ócio nos horários de $8 \mathrm{~h}, 10 \mathrm{~h}$ e $16 \mathrm{~h}$. Os horários de $12 \mathrm{~h}$ e $14 \mathrm{~h}$ apresentaram-se como os mais estressantes termicamente com base na expressão de maiores movimentos de desconforto e nos índices térmicos encontrados.

O comportamento "Comendo" foi observado com um maior número de aves na parte da manhã. Isso pode ter ocorrido em virtude do manejo realizado na alimentação, em que as aves recebiam ração no final da tarde, tendo ocorrido ainda pela manhã a procura do alimento no comedouro. Os comportamentos "Forragear", "Explorando Penas" e "Bicagem Não Agressiva" foram mais frequentes às $8 \mathrm{~h}$ e $16 \mathrm{~h}$, quando as temperaturas e os índices de conforto térmico apresentaram menores valores, portanto, horários mais propícios para estes comportamentos acontecerem. Estes resultados concordam em parte com os resultados encontrados por Alves (2006), que observou maior número de poedeiras criadas em piso forrageando e explorando penas nos horários mais frescos do dia. Porém, o autor não observou o comportamento "Bicagem Não Agressiva".

O comportamento "Bicagem Agressiva" foi observado com maior incidência às $16 \mathrm{~h}$, e nos demais horários não diferiram entre si. O comportamento "Ciscando" ocorreu com maior frequência às $8 \mathrm{~h}$, por ser o horário em que as aves eram soltas do abrigo. Os horários das $10 \mathrm{~h}$ e $16 \mathrm{~h}$ não diferiram entre si, mas diferiram dos horários das $12 \mathrm{~h}$ e $14 \mathrm{~h}$, que foram iguais estatisticamente. Nos horários das $8 \mathrm{~h}, 10 \mathrm{~h}$ e $16 \mathrm{~h}$, os índices de conforto térmico estiveram mais baixos que os das $12 \mathrm{~h}$ e $14 \mathrm{~h}$, o que pode ter interferido na quantidade de aves ciscando. Segundo Barbosa Filho et al. (2007), o ato de ciscar é um comportamento característico e natural das aves, caracterizado como movimentos de exploração do território feitos pelos pés e bicos; porém, em condições de altas temperaturas, estes movimentos diminuem.

\section{Conclusão}

As linhagens Pescoço Pelado (Label Rouge), Caipira Vermelho Pesadão, Carijó, Master Griss e Colorido apresentam desconforto térmico, mas não apresentam diferenças no seu desempenho e na sua fisiologia, em sistema semi-intensivo para regiões de clima quente.

\section{Agradecimentos}

À Fundação de Amparo à Pesquisa do Estado de Minas Gerais (Fapemig), pelo apoio financeiro.

\section{Referências}

ALVES, S.P. Uso da zootecnia de precisão na avaliação do bemestar bioclimático de aves poedeiras em diferentes sistemas de criação. 2006. 128p. Tese (Doutorado) - Universidade de São Paulo, Piracicaba.

BARBOSA FILHO, J.A.D.; SILVA, I.J.O.; SILVA, M.A.N.; SILVA, C.J.M. Avaliação dos comportamentos de aves poedeiras utilizando sequência de imagens. Engenharia Agrícola, v.27, p.93-99, 2007. DOI: 10.1590/S0100-69162007000100002.

BUFFINGTON, D.E.; COLLAZO-AROCHO, A.; CANTON, G.H.; PITT, D.; THATCHER, W.W.; COLLIER, R.J. Black globe-humidity index (BGHI) as comfort equation for dairy 
cows. Transaction of the ASAE, v.24, p.711-714, 1981. DOI: $10.13031 / 2013.34325$.

CANGAR, $\ddot{\boldsymbol{O}}$; AERTS, J.-M.; BUYSE, J.; BERCKMANS, D. Quantification of the spatial distribution of surface temperatures of broilers. Poultry Science, v.87, p.2493-2499, 2008. DOI: 10.3382/ps.2007-00326.

CARVALHO, G.B. de; LOPES, J.B.; SANTOS, N.P. da S.; REIS, N.B. do N.; CARVALHO, W.F. de; SILVA, S.F.; CARVALHO, D.A. de; SILVA, E.M. da; SILVA, S.M. da. Comportamento de frangos de corte criados em condições de estresse térmico alimentados com dietas contendo diferentes níveis de selênio. Revista Brasileira de Saúde e Produção Animal, v.14, p.785-797, 2013. DOI: $10.1590 /$ S1519-99402013000400012.

CORDEIRO, M.B.; FREITAS, H.J. de; AQUINO, E.O.; SOUSA, E.M. Avaliação do estresse térmico em frangos caipiras criados em condições climáticas do Estado do Acre. Enciclopédia Biosfera, v.10, p.358-365, 2014.

DEL CASTILHO, C.C.; SANTOS, T.T; RODRIGUES, C.A.F.; TORRES FILHO, R.A. Effects of sex and genotype on performance and yield characteristics of free range broiler chickens. Arquivo Brasileiro de Medicina Veterinária e Zootecnia, v.65, p.1483-1490, 2013. DOI: 10.1590/S0102-09352013000500029.

DIXON, L.M.; DUNCAN, I.J.H.; MASON, G.J. The effects of four types of enrichment on feather-pecking behavior in laying hens housed in barren environments. Animal Welfare, v.19, p.429-435, 2010.

FANATICO, A.C.; PILLAI, P.B.; EMMERT, J.L.; OWENS, C.M. Meat quality of slow-and fast-growing chicken genotypes fed low-nutrient of standard diets and raised indoors or with outdoor access. Poultry Science, v.86, p.2245-2255, 2007. DOI: 10.1093/ ps/86.10.2245.

FARIA, P.B.; BRESSAN, M.C.; SOUZA, X.R. de; RODRIGUES, E.C.; CARDOSO, G.P.; GAMA, L.T. da. Composição proximal e qualidade da carne de frangos das linhagens Paraíso Pedrês e Pescoço Pelado. Revista Brasileira de Zootecnia, v.38, p.24552464, 2009. DOI: 10.1590/S1516-35982009001200023.

FOUAD, M.A.; RAZEK, A.H.A.; BADAWY, E.S.M. Broilers welfare and economics under two management alternatives on commercial scale. International Journal of Poultry Science, v.7, p.1167-1173, 2008. DOI: 10.3923/ijps.2008.1167.1173.

FRASER, D.; DUNCAN, I.J.H.; EDWARDS, S.A.; GRANDIN, T.; GREGORY, N.G.; GUYONNET, V.; HEMSWORTH, P.H.; HUERTAS, S.M.; HUZZEY, J.M.; MELLOR, D.J.; MENCH, J.A.; SPINKA, M.; WHAY, H.R. General principles for the welfare of animals in production systems: the underlying science and its application. The Veterinary Journal, v.198, p.19-27, 2013. DOI: $10.1016 /$ j.tvj1.2013.06.028.

GONÇALVES, S.A. Comportamento de diferentes linhagens de frango de corte tipo caipira. 2012. 35p. Dissertação (Mestrado) - Universidade Federal dos Vales do Jequitinhonha e Mucuri, Diamantina.

LARA, L.J.; ROSTAGNO, M.H. Impact of heat stress on poultry production. Animals, v.3, p.356-369, 2013. DOI: 10.3390/ ani3020356.
LU, Q.; WEN, J.; ZHANG, H. Effect of chronic heat exposure on fat deposition and meat quality in two genetic types of chicken. Poultry Science, v.86, p.1059-1064, 2007. DOI: 10.1093/ ps/86.6.1059.

MACK, L.A.; FELVER-GANT, J.N.; DENNIS, R.L.; CHENG, H.W. Genetic variations alter production and behavioral responses following heat stress in 2 strains of laying hens. Poultry Science, v.92, p.285-294, 2013. DOI: 10.3382/ps.2012-02589.

MADEIRA, L.A.; SARTORI, J.R.; ARAÚJO, P.C.; PIZZOLANTE, C.C.; SALDANHA, E.S.P.B.; PEZZATO, A.C. Avaliação do desempenho e do rendimento de carcaça de quatro linhagens de frangos de corte em dois sistemas de criação. RevistaBrasileira de Zootecnia, v.39, p.2214-2221, 2010.

MARTIN, P.; BATESON, P. Measuring Behaviour: an introductory guide. Cambridge: University Press, 1986. 200p.

MEDEIROS, C.M.; BAETA, F.C.; OLIVEIRA, R.F.M.; TINOCO, I.F.F.; ALBINO, L.F.T.: CECON, P.R. Efeito da temperatura, umidade relativa e velocidade do ar em frangos de corte. Engenharia na Agricultura, v.13, p.277-286, 2005.

MIKULSKI, D.; CELEJ, J.; JANKOWSKI, J.; MAJEWSKA, T.; MIKULSKA, M. Growth performance, carcass traits and meat quality of slower-growing and fast-growing chickens raised with and without outdoor access. Asian-Australasian Journal of Animal Sciences, v.24, p.1407-1416, 2011. DOI: 10.5713/ ajas.2011.11038.

MOURA, D.J.; BUENO, L.G.F.; LIMA, K.A.O.; CARVALHO, T.M.R.; MAIA, A.P.A.M. Strategies and facilities in order to improve animal welfare. Revista Brasileira de Zootecnia, v.39, p.311-316, 2010. Suplemento especial. DOI: 10.1590/S151635982010001300034.

NAZARENO, A.C.; PANDORFI, H.; GUISELINI, C.; VIGODERIS, R.B.; PEDROSA, E.M.R. Bem-estar na produção de frango de corte em diferentes sistemas de criação. Engenharia Agrícola, v.31, p.13-22, 2011. DOI: 10.1590/S010069162011000100002.

PEREIRA, D.F.; SALGADO, D.D.; NÄÄS, I.A.; PENHA, N.L.J.; BIGHI, C.A. Efeitos da temperatura do ar, linhagem e período do dia nas frequências de ocorrências e tempos de expressão comportamental de matrizes pesadas. Engenharia Agrícola, v.27, p.596-610, 2007. DOI: 10.1590/S0100-69162007000400002.

SANTOS, M.J.B. dos; PANDORFI, H.; ALMEIDA, G.L.P.; MORRIL, W.B.; PEDROSA, E.M.R.; GUISELINI, C. Comportamento bioclimático de frangos de corte caipira em piquetes enriquecidos. Revista Brasileira de Engenharia Agrícola e Ambiental, v.14, p.554-560, 2010.

SAVINO, V.J.M.; COELHO, A.A.D.; ROSÁRIO, M.F. do; SILVA, M.A.N. da. Avaliação de materiais genéticos visando à produção de frango caipira em diferentes sistemas de alimentação. Revista Brasileira de Zootecnia, v.36, p.578-583, 2007. DOI: 10.1590/ S1516-35982007000300009.

SILVA, I.J.O. da; BARBOSA FILHO, J.A.D.B.; SILVA, M.A.N. da; PIEDADE, S.M. de S. Influência do sistema de criação nos parâmetros comportamentais de duas linhagens de poedeiras submetidas a duas condições ambientais. Revista Brasileira Zootecnia, v.35, p.1439-1446, 2006. 
SILVA, M.A.N.; SILVA, I.J.O.; PIEDADE, S.M.S.; MARTINS, E.; COELHO, A.A.D.; SAVINO, V.J.M. Resistência ao estresse calórico em frangos de corte de pescoço pelado. Revista Brasileira de Ciência Avícola, v.3, p.1-8, 2001. DOI: 10.1590/ S1516-635X2001000100002.

SILVA, M.A.N. da; HELLMEISTER FILHO, P.; ROSÁRIO, M.F. do; COLEHO, A.A.D.; SAVINO, V.J.M.; GARCIA, A.A.F.; SILVA, I.J.O. da; MENTEN, J.F.M. Influência do sistema de criação sobre o desempenho, a condição fisiológica e o comportamento de linhagens de frangos para corte. Revista Brasileira de Zootecnia, v.32, p.208-213, 2003.

SILVA, R.G. Introdução à bioclimatologia animal. São Paulo: Nobel, 2000. 286p.

SOLEIMANI, A.F.; ZULKIFLI, I.; OMAR, A.R.; RAHA, A.R. Physiological responses of 3 chicken breeds to acute heat stress. Poultry Science, v.90, p.1435-1440, 2011. DOI: 10.3382/ps.201101381.
TAKAHASHI, S.E.; MENDES, A.A.; SALDANHA, E.S.P.B.; PIZZOLANTE, C.C.; PELÍCIA, K.; GARCIA, R.G.; PAZ, I.C.L.A.; QUINTEIRO, R.R. Efeito do sistema de criação sobre o desempenho e rendimento de carcaça de frangos de corte tipo colonial. Arquivo Brasileiro de Medicina Veterinária e Zootecnia, v.58, p.624-632, 2006. DOI: 10.1590/S010209352006000400026.

TAN, G.-Y.; YANG, L.; FU, Y.-Q.; FENG, J.-H.; ZHANG, M.-H. Effects of different acute high ambient temperatures on function of hepatic mitochondrial respiration, antioxidative enzymes, and oxidative injury in broiler chickens. Poultry Science, v.89, p.115122, 2010. DOI: 10.3382/ps.2009-00318.

VELOSO, R.C.; PIRES, A.V.; TORRES FILHO, R.A.; PINHEIRO, S.R.F.; WINKELSTROTER, L.K.; ALCÂNTARA, D.C.; CRUZ, C.C.D.C.S. Parâmetros de desempenho e carcaça de genótipos de frangos tipo caipira. Arquivo Brasileiro de Medicina Veterinária e Zootecnia, v.66, p.1251-1259, 2014. DOI: $10.1590 / 1678-6312$.

Recebido em 25 de agosto de 2015 e aprovado em 25 de agosto de 2016 